\title{
Selamento superficial e formação de crosta em solos representativos do Estado da Paraíba
}

Rodolpho José de Almeida Nascimento, Flávio Pereira de Oliveira, Djail Santos, Raphael Moreira Beirigo, Paulo Roberto Megna Francisco, Raiff Ramos de Almeida Nascimento

Universidade Federal da Paraíba - UFPB, Centro de Ciências Agrárias - CCA, Areia - PB. E-mail: rjan85@gmail.com

\section{Resumo}

A formação de selo e crosta superficial no solo tem consequências diretas para o desenvolvimento das culturas e qualidade ambiental, alterando sua estrutura, diminuindo a disponibilidade de água para as plantas e potencializando os efeitos da erosão. Diante disto, o presente trabalho teve como objetivo avaliar as alterações causadas por sucessivos eventos de precipitação na superfície de solos. Foram avaliadas quatro classes de solos: Latossolo Amarelo (LA); Argissolo Vermelho (PV); Neossolo Litólico (RL); Luvissolo Crômico (TC). O critério de seleção teve como base o grau de desenvolvimento pedogenético. $O$ experimento foi conduzido sob chuva simulada, em parcelas experimentais com área útil de 0,3481 $\mathrm{m}^{2}$, com solo descoberto e declividade de $0,10 \mathrm{~mm}^{-1}$. Os testes foram realizados com intensidade média da precipitação ajustada em $85 \mathrm{~mm} \mathrm{~h}^{-1}$ durante 75 minutos, aplicadas em três eventos consecutivos com intervalos de 7 dias, para determinar os efeitos da formação do selamento superficial durante a chuva e a formação de crosta após o período de secagem do solo. Foram avaliadas as variáveis hidráulicas do escoamento, a perda de solo (PSi), taxa de desagregação do solo em entressulcos $\left(D_{i}\right)$ e o fator de erodibilidade em entressulcos $\left(K_{i}\right)$. A formação de selamento e de crostas superficiais foram observadas com aumento da velocidade de escoamento, da descarga liquida e a diminuição da altura da lâmina de água, assim como alterações em $P S i, D_{i}$ e $K_{i}$, sendo no primeiro evento de chuva simulada a maior contribuição para formação do encrostamento superficial. O TC se mostrou o mais estável após os eventos sucessivos de chuva simulada, mesmo tendo a rugosidade superficial modificada, não apresentou alterações significativas nas perdas de solo, taxa de desagregação e erodibilidade do solo em entresulco.

Palavras - chave: concentração de sedimentos; erosão entressulcos; rugosidade superficial; semiárido; variáveis hidráulicas do escoamento.

\section{Surface sealing and crust formation in Paraíba State representative soils}

\begin{abstract}
The formation of seal and surface crust in the soil has direct consequences for the development of the crops and environmental quality, altering the soil structure, reducing the availability of water to the plants and favoring the erosion. The objective of this study was to evaluate the changes caused by successive rainfall events on the soil surface. Four soils were evaluated: Ferralsol (FR); Acrisol (AC); Leptosol (LP); Luvisol (LV). The selection criterion was based on pedogenetic development. The experiment was conducted under simulated rainfall, in experimental plots with a useful area of $0,3481 \mathrm{~m}^{2}$, uncovered soil and slope of $0.10 \mathrm{~mm}^{-1}$. The tests were performed with mean precipitation intensity of $85 \mathrm{~mm} \mathrm{~h}^{-1}$ for 75 min, applied in three consecutive events at 7 days intervals, to determine the effects of surface sealing during rainfall and crust formation after the soil drying period. The hydraulic flow variables, the soil losses (PSi), soil disaggregation rate in interrill $\left(D_{i}\right)$ and the interrill erodibility factor $\left(K_{i}\right)$ were evaluated. The formation of sealing and surface crusts were observed with increase of the flow velocity and liquid discharge, and the decrease of water sheet height, as well as in the changes in PSi, $D_{i}$ and $K_{i}$, being in the first event of simulated rainfall the greater contribution to formation of superficial crusting. LV showed the most stable after the successive events of simulated rainfall, even though the surface roughness was modified, did not present significant changes in the soil losses, disaggregation rate and soil interrill erodibility.
\end{abstract}

Keywords: sediment concentration; interrill erosion; ; surface roughness; semiarid; hydraulic flow variables. 


\section{Introdução}

A ação direta do impacto das gotas de chuva na superfície do solo descoberto ocasiona uma série de efeitos: desintegração de agregados, destacamento, transporte e deposição de partículas, causando erosão hídrica e modificando a estrutura superficial do solo de várias formas. Uma das alterações que ocorre é a formação de encrostamento superficial, característica comum de solos cultivados em várias regiões do mundo (KINNELL, 2005; HU et al., 2012; DALLA ROSA et al., 2013). A formação de crostas superficiais no solo tem consequências diretas para o desenvolvimento das culturas e para a qualidade ambiental, pois reduz a infiltração de água no solo e a disponibilidade de água para as raízes das plantas, dificulta a emergência de plântulas devido à compactação da camada superficial do solo, aumenta o escoamento superficial e a erosão hídrica (ASSOULINE, 2004), reduz a produção agrícola e provoca impactos ambientais, como a contaminação de solo e da água através do transporte de insumos agrícolas, e o assoreamento de cursos d'água e reservatórios pela deposição de partículas do solo oriundas do destacamento e transporte pela ação do fluxo de água (TOUMA et al., 2011; VAEZl; BAHRAMI, 2014; MONTANARELLA et al., 2016).

A porosidade na superfície do solo é significativamente reduzida devido ao preenchimento e entupimento dos poros pelo material fino transportado pela enxurrada, a compactação associada aos impactos de gotas de chuva e a deposição de partículas de argila na superfície do solo após chuva (ASSOULINE, 2004). Esse processo dá origem a formação de um selo estrutural. A desidratação do selo superficial causado pela chuva resulta na formação de uma crosta estrutural, ou seja, o selamento superficial ocorre na fase úmida ou durante a precipitação, e a crosta superficial se forma a partir da secagem do solo, tornando o selo superficial em crosta superficial (ARMENISE et al., 2018).

Durante o processo de formação do selo superficial, as partículas do solo são rearranjadas e consolidadas em uma estrutura coesa, cuja espessura pode variar de 0,1 a $50 \mathrm{~mm}$, modificando as propriedades físicas do solo, principalmente a condutividade hidráulica, devido a redução no número e na continuidade do espaço poroso, afetando a infiltração e a distribuição de água ao logo do perfil do solo, além de alterar a rugosidade superficial do solo ao mesmo tempo que aumenta o escoamento superficial e a erosão por salpicamento (CARMI; BERLINER, 2008; HU et al., 2012; ZONTA et al., 2012; ARMENISE et al., 2018).

As alterações das condições superficiais dependem das características do solo como textura, mineralogia, matéria orgânica, umidade inicial, distribuição, tamanho e estabilidade dos agregados, entre outros (LE BISSONNAIS; SINGER, 1993; FOX; LE BISSONNAIS, 1998), bem como dos fatores climáticos, especialmente da chuva (intensidade, distribuição de tamanho de gota, energia cinética), além da cobertura vegetal, atividade biológica e uso da terra (LE BISSONNAIS et al., 2005)

Segundo Dalla Rosa et al. (2013) e Brandão et al. (2006), o estudo e o detalhamento do processo de formação de crostas do solo podem ser monitorados diretamente, utilizando técnicas de microscopia para análises micromorfológicas, ou indiretamente, pela observação da alteração nas características físicas e hídricas do solo inerentes a condutividade hidráulica e a infiltração de água no solo. Diversos estudos abordando selamento superficial e encrostamento do solo têm sido realizados desde o início do século $X X$, e o interesse em ambos os fenômenos, não surpreendentemente, continua, em razão das suas importantes consequências, no meio ambiente, além de ocorrência do fenômeno em culturas com importância econômica (SOUZA et al., 2014).

Devido às particularidades de cada classe de solo, e as variações existentes dentro de cada classe por diferenças edafoclimáticas no ambientes de formação, e ainda, pela importância socioeconômica que cada uma das classes avaliadas representa para as diferentes regiões, faz-se necessários estudos sobre a erosão hídrica e suas consequências para as particularidades locais visando obtenção do conhecimento sobre os processos envolvidos na formação do selo e da crosta superficial para escolha das melhores estratégias para preservação ambiental e da capacidade produtiva sustentável do solo.

Para testar a hipótese de que solos com distintos estágios de desenvolvimento pedogenético respondem de maneira diferente ao selamento superficial e a posterior formação de crosta superficial, o presente trabalho teve como objetivo avaliar por metodologia indireta: I) as alterações causadas pela erosão hídrica na 
superfície do solo representada pelo coeficiente de rugosidade de Manning; II) as mudanças nas características das variáveis hidráulicas como a velocidade média, a altura da lâmina e a descarga líquida unitária do escoamento; III) e o fator de erodibilidade em entressulcos de quatro solos representativos do estado da Paraíba, em três períodos de aplicação de chuva simulada.
Foram utilizadas amostras de quatro solos que fazem parte do Banco de Solos Representativos do Estado da Paraíba (OLIVEIRA et al., 2006), selecionados de acordo com as diferentes classes, sua representatividade e grau de desenvolvimento pedogenético (Tabela 1), sendo eles: Latossolo Amarelo (LA), Argissolo Vermelho (PV), Neossolo Litólico (RL) e Luvissolo Crômico (TC).

\section{Material e Métodos}

Tabela 1. Classes de solo, localização de coleta, vegetação predominante, relevo, média de precipitação anual, formação geológica e litologia, mineralogia e índice de intemperismo dos solos avaliados

\begin{tabular}{|c|c|c|c|c|}
\hline \multirow{3}{*}{ Variáveis } & \multicolumn{4}{|c|}{ Solos ${ }^{(1)}$} \\
\hline & \multicolumn{2}{|c|}{ Mais desenvolvidos ${ }^{(6)}$} & \multicolumn{2}{|c|}{ Menos desenvolvidos ${ }^{(6)}$} \\
\hline & LA & PV & $\mathbf{R L}$ & TC \\
\hline Perfil (2) & 4 & 23 & 53 & 30 \\
\hline U.M. ${ }^{(2)}$ & LVe1 & Tre1 & SS2 & NC4 \\
\hline $\begin{array}{c}\text { Microrregião } \\
\text { Geográfica / Cidade }\end{array}$ & $\begin{array}{l}\text { Curimataú } \\
\text { Ocidental / } \\
\text { Cuité }\end{array}$ & $\begin{array}{c}\text { Guarabira / Alagoa } \\
\text { Grande }\end{array}$ & $\begin{array}{c}\text { Curimataú Ocidental / } \\
\text { Pocinhos }\end{array}$ & $\begin{array}{c}\text { Sapé / São Miguel de } \\
\text { Taipú }\end{array}$ \\
\hline Vegetação $^{(2)}$ & $\begin{array}{c}\text { Floresta } \\
\text { Subcaducifólia }\end{array}$ & $\begin{array}{c}\text { Floresta } \\
\text { Subcaducifólia }\end{array}$ & Caatinga Hiperxerófila & Floresta Caducifólia \\
\hline Relevo ${ }^{(2)}$ & Plano & Ondulado & $\begin{array}{c}\text { Plano a suavemente } \\
\text { ondulado }\end{array}$ & Ondulado \\
\hline $\begin{array}{c}\text { Precipitação e } \\
\text { Concentração das } \\
\text { chuvas }^{(4)}\end{array}$ & $\begin{array}{c}872,7 \mathrm{~mm} \\
\text { Março a Maio }\end{array}$ & $\begin{array}{c}950,0 \mathrm{~mm} \\
\text { Março a Agosto }\end{array}$ & $\begin{array}{c}382,3 \mathrm{~mm} \\
\text { Março a Julho }\end{array}$ & $\begin{array}{l}\text { 1029,3 mm } \\
\text { Março a Julho }\end{array}$ \\
\hline $\begin{array}{l}\text { Classificação de } \\
\text { Köppen }\end{array}$ & As & BSh & BSh & As \\
\hline $\begin{array}{l}\text { Formação geológica e } \\
\text { litologia }^{(2)}\end{array}$ & $\begin{array}{l}\text { Terciário / } \\
\text { Sedimentos da } \\
\text { Série Serra do } \\
\text { Martins }\end{array}$ & $\begin{array}{c}\text { Pré-Cambiano (CD) } \\
\text { / Hornblenda- } \\
\text { biotita-gnaisse. }\end{array}$ & $\begin{array}{l}\text { Pré-Cambiano / } \\
\text { Gnaisse. }\end{array}$ & $\begin{array}{l}\text { Pré-Cambiano (CD) / } \\
\text { Hornblenda-gnaisse. }\end{array}$ \\
\hline Minerais ${ }^{(3)}$ & $\mathrm{Ct}, \mathrm{Gt}$ & $\mathrm{Ct}, \mathrm{Gt}, \mathrm{Mi}, \mathrm{Hm}$ & $\mathrm{Mi}, \mathrm{Ct},(2: 1)$ & $\mathrm{Ct}, \mathrm{Mi},(2: 1), \mathrm{Fp}$ \\
\hline $\mathrm{Ki}\left(\mathrm{SiO}_{2} / \mathrm{Al}_{2} \mathrm{O}_{3}\right)^{(2)}$ & 2,16 & 2,25 & 2,93 & 3,42 \\
\hline
\end{tabular}

Fontes: ${ }^{(1)}$ De acordo com o Sistema Brasileiro de Classificação do Solo (EMBRAPA, 2013); ${ }^{(2)}$ Brasil (1972); ${ }^{(3)}$ Dados apresentados por Farias et al. (2009) na ordem de sua predominância na fração argila do solo; ${ }^{(4)}$ Dados obtidos pela Agência Executiva de Gestão das Águas do Estado da Paraíba - AESA (2019); ${ }^{(5)}$ Classificação realizada por Francisco et al. (2015). Legenda: U.M.= Unidade de Mapeamento; $\mathrm{Ki}\left(\mathrm{SiO}_{2} / \mathrm{Al}_{2} \mathrm{O}_{3}\right)=$ Índice de intemperismo; $\mathrm{LA}=$ Latossolo Amarelo; PV = Argissolo Vermelho; $\mathrm{RL}=$ Neossolo Litólico; $\mathrm{TC}=$ Luvissolo Crômico; $\mathrm{Ct}=$ caulinita; $\mathrm{Gt}=$ goethita; $\mathrm{Mi}=$ mica; $\mathrm{Hm}=$ hematita; $(2: 1)=$ argilomineral tipo $2: 1 ; \mathrm{Fp}=$ feldspatos; ${ }^{(6)}$ Solos mais desenvolvidos: $\mathrm{Ki} \leq 2,46$; Solos menos desenvolvidos: $\mathrm{Ki}>2,46$.

A caracterização física e química dos solos avaliados (Tabela 2) foi realizada seguindo a metodologia proposta por Teixeira et al. (2017). Além do índice de estabilidade de agregados proposto na metodologia citada (IEA $\mathrm{T}_{\mathrm{T}}$ ), também foi determinado índice de estabilidade de agregados (IEA $A_{c}$ ) através da equação proposta por Cesário et al. (2010), desenvolvida a partir da análise crítica das equações propostas por Castro Filho (1998) e Hillel (2003): 
Tabela 2. Características físicas e químicas dos solos avaliados

\begin{tabular}{|c|c|c|c|c|}
\hline \multirow{3}{*}{ Características } & \multicolumn{4}{|c|}{ Solos ${ }^{(1)}$} \\
\hline & \multicolumn{2}{|c|}{ Mais desenvolvidos ${ }^{(5)}$} & \multicolumn{2}{|c|}{ Menos desenvolvidos ${ }^{(5)}$} \\
\hline & LA & PV & $\mathbf{R L}$ & TC \\
\hline \multicolumn{5}{|l|}{ Físicas $^{(2)}$} \\
\hline Areia Total $\left(\mathrm{g} \mathrm{kg}^{-1}\right)$ & 627 & 548 & 795 & 798 \\
\hline Areia muito grossa $\left(\mathrm{g} \mathrm{kg}^{-1}\right)$ & 27 & 18 & 19 & 133 \\
\hline Areia grossa $\left(\mathrm{g} \mathrm{kg}^{-1}\right)$ & 110 & 54 & 135 & 242 \\
\hline Areia média $\left(\mathrm{g} \mathrm{kg}^{-1}\right)$ & 223 & 128 & 256 & 206 \\
\hline Areia fina $\left(\mathrm{g} \mathrm{kg}^{-1}\right)$ & 218 & 263 & 283 & 160 \\
\hline Areia muito fina $\left(\mathrm{g} \mathrm{kg}^{-1}\right)$ & 49 & 85 & 102 & 57 \\
\hline Silte $\left(\mathrm{g} \mathrm{kg}^{-1}\right)$ & 188 & 124 & 120 & 102 \\
\hline Argila $\left(\mathrm{g} \mathrm{kg}^{-1}\right)$ & 185 & 328 & 85 & 100 \\
\hline Argila Dispersa H2O $\left(\mathrm{g} \mathrm{kg}^{-1}\right)$ & 34,6 & 45,1 & 20,4 & 23,7 \\
\hline Grau de Floculação (\%) & 81 & 86 & 76 & 76 \\
\hline Densidade do Solo $\left(\mathrm{kg} \mathrm{dm}^{-3}\right)$ & 1,42 & 1,46 & 1,62 & 1,51 \\
\hline Densidade de Partículas $\left(\mathrm{kg} \mathrm{dm}^{-3}\right)$ & 2,52 & 2,58 & 2,44 & 2,63 \\
\hline Porosidade Total $\left(\mathrm{m}^{3} \mathrm{~m}^{-3}\right)$ & 0,44 & 0,43 & 0,34 & 0,43 \\
\hline $\mathrm{DMPA}_{U}$ & 1,11 & 0,63 & 0,44 & 1,66 \\
\hline $\mathrm{DMPA}_{S}$ & 1,76 & 2,57 & 1,19 & 1,89 \\
\hline $\mathrm{IEA}_{\mathrm{T}}(\%)$ & 63,4 & 24,5 & 37,3 & 87,7 \\
\hline $\mathrm{IEA}_{c}(\%)$ & 62,1 & 33,9 & 20,4 & 64,1 \\
\hline \multicolumn{5}{|l|}{ Químicas $^{(3)}$} \\
\hline $\mathrm{pH}\left(\mathrm{H}_{2} \mathrm{O}\right)$ & 5,9 & 5,0 & 6,2 & 6,2 \\
\hline C.O. $\left(\mathrm{g} \mathrm{kg}^{-1}\right)$ & 5,9 & 8,0 & 3,8 & 7,6 \\
\hline$P\left(\mathrm{mg} \mathrm{dm}^{-3}\right)$ & 11,42 & 2,32 & 4,31 & 4,35 \\
\hline $\mathrm{K}\left(\mathrm{cmol}_{\mathrm{c}} \mathrm{dm}^{-3}\right)$ & 0,18 & 0,19 & 0,19 & 0,64 \\
\hline $\mathrm{Ca}^{2+}\left(\mathrm{cmol}_{\mathrm{c}} \mathrm{dm}^{-3}\right)$ & 1,6 & 1,9 & 2 & 6,1 \\
\hline $\mathrm{Mg}^{2+}\left(\mathrm{cmol}_{\mathrm{c}} \mathrm{dm}^{-3}\right)$ & 1,15 & 0,80 & 1,10 & 4,00 \\
\hline $\mathrm{Na}^{+}\left(\mathrm{cmol}_{\mathrm{c}} \mathrm{dm}^{-3}\right)$ & 0,11 & 0,04 & 0,25 & 0,10 \\
\hline $\mathrm{Al}^{3+}\left(\mathrm{cmol}_{\mathrm{c}} \mathrm{dm}^{-3}\right)$ & 0,00 & 0,32 & 0,05 & 0,00 \\
\hline $\mathrm{H}+\mathrm{Al}\left(\mathrm{cmol}_{\mathrm{c}} \mathrm{dm}^{-3}\right)$ & 2,14 & 4,28 & 2,29 & 2,90 \\
\hline СТC pH 7,0 $\left(\mathrm{cmol}_{\mathrm{c}} \mathrm{dm}^{-3}\right)$ & 5,18 & 7,20 & 5,83 & 13,74 \\
\hline Saturação por Bases (V \%) & 59 & 41 & 61 & 79 \\
\hline Saturação por Alumínio (m \%) & 0 & 10 & 1 & 0 \\
\hline $\mathrm{Fe}_{\mathrm{o}}\left(\mathrm{g} \mathrm{kg}^{-1}\right)^{(4)}$ & 0,25 & 0,25 & 0,66 & 0,79 \\
\hline $\mathrm{Fe}_{\mathrm{d}}\left(\mathrm{g} \mathrm{kg}^{-1}\right)^{(4)}$ & 7,63 & 7,63 & 2,13 & 4,69 \\
\hline $\mathrm{Al}_{\circ}\left(\mathrm{g} \mathrm{kg}^{-1}\right)^{(4)}$ & 0,33 & 0,33 & 0,16 & 0,33 \\
\hline$A l_{d}\left(g_{k g}^{-1}\right)^{(4)}$ & 1,12 & 1,12 & 0,21 & 0,38 \\
\hline
\end{tabular}

Fontes: ${ }^{(1)}$ De acordo com o Sistema Brasileiro de Classificação do Solo (EMBRAPA, 2013); ${ }^{(2)}$ Obtido a partir das bases de dados apresentadas por Pequeno (2016) para LA, PV e RL, e por Freitas (2016) para TC; ${ }^{(3)}$ Dados apresentados por Farias et al. (2009); ${ }^{(4)}$ Dados apresentados por Santos et al. (2011). Legenda: LA = Latossolo Amarelo; PV = Argissolo Vermelho; $\mathrm{RL}=$ Neossolo Litólico; $\mathrm{TC}=$ Luvissolo Crômico; Areia Total $(2-0,053 \mathrm{~mm}) ; \mathrm{AMG}=$ Areia muito grossa $(2-1$ $\mathrm{mm}) ; A G=$ Areia grossa $(1-0,5 \mathrm{~mm}) ; A M=$ Areia média $(0,5-0,25 \mathrm{~mm}) ; A F=$ Areia fina $(0,25-0,106 \mathrm{~mm}) ; A M F=$ Areia muito fina (0,106-0,053 mm); Silte (0,053-0,002 mm); Argila $(<0,002 \mathrm{~mm}) ; \mathrm{DMPA}_{U}=$ Diâmetro médio ponderado dos agregados obtidos via peneiragem úmida; $\mathrm{DMPA}_{\mathrm{s}}=$ Diâmetro médio ponderado dos agregados obtidos via peneiragem seca; IEA ${ }_{T}$ = Índice de estabilidade de agregados segundo metodologia proposta por Teixeira et al. (2017); $\mathrm{IEA}_{c}=$ Índice de estabilidade de agregados segundo metodologia proposta por Cesário et al. (2010); C.O. = Carbono Orgânico; $\mathrm{Fe}_{\mathrm{o}}$ e $\mathrm{Al}_{\mathrm{o}}=$ Óxidos de ferro e alumínio extraídos com oxalato de amônio ácido; $\mathrm{Fe}_{\mathrm{d}}$ e $\mathrm{Al}_{\mathrm{d}}=$ Óxidos de ferro e alumínio extraído com ditionito-citrato-bicarbonato. ${ }^{(5)}$ Solos mais desenvolvidos: $\mathrm{Ki} \leq 2,46$; Solos menos desenvolvidos: $\mathrm{Ki}>2,46$. Médias seguidas de mesmas letras, em linhas, não diferem entre si pelo teste de Tukey a $5 \%$ de probabilidade. 
A erosão hídrica dos solos em entressulcos foi determinada em laboratório, sob chuva simulada, em solo descoberto. As amostras dos solos foram coletadas em campo, na camada superficial de $0-20 \mathrm{~cm}$ de profundidade, em condições de conservação mais próximas as condições naturais, ou sem cultivo agrícola. Posteriormente, foram destorroados, passadas em peneiras com abertura de malha de $10 \mathrm{~mm}$, e postas para secagem ao ar.

As parcelas experimentais foram construídas com material em acrílico e montadas sobre uma estrutura metálica, com área útil de $0,3481 \mathrm{~m}^{2}$, com dimensões de $0,59 \mathrm{~m}$ por $0,59 \mathrm{~m}$ e altura de $0,10 \mathrm{~m}$, com bordadura lateral de 0,20 $\mathrm{m}$ de largura. $\mathrm{O}$ volume da parcela experimental foi preenchido com uma camada de $3 \mathrm{~cm}$ de espessura de brita, sobre a qual foi colocada uma camada de $1 \mathrm{~cm}$ de areia com diâmetro $0,25 \mathrm{~mm}$, uma tela de plástico com malha de $1 \mathrm{~mm}$ e uma camada de solo com $6 \mathrm{~cm}$ de espessura, para uma densidade de empacotamento (Dep) de 1,2 $\mathrm{g} \mathrm{cm}^{-3}$.

As amostras de solo nas parcelas foram saturadas com antecedência de 24 horas, mediante um conjunto de seis mangueiras acopladas ao fundo da parcela, conectadas a um reservatório de água posicionado a uma altura levemente superior ao topo da parcela experimental. Ao iniciar os testes com chuva, aplicou-se uma tensão de água equivalente a 60 $\mathrm{cm}$ de coluna d'água (60 cca), a qual foi mantida durante a aplicação do ensaio. A declividade das parcelas experimentais foi de $0,10 \mathrm{~m} \mathrm{~m}^{-1}$. As chuvas simuladas foram efetuadas utilizando-se simulador de chuvas, semelhante ao descrito por Meyer e Harmon (1979), com um bico aspersor tipo Veejet 80-100 com diâmetro interno de 12,7 $\mathrm{mm}$ situado a 3,1 $\mathrm{m}$ acima da superfície da parcela, operando com uma pressão constante de $41 \mathrm{kPa}$ na saída de água do bico. Em todos os ensaios, foram aplicadas chuvas simuladas com três repetições para cada solo, com intensidade média ajustada em $85 \mathrm{~mm} \mathrm{~h}^{-1}$ durante 75 minutos.

As intensidades médias das chuvas foram determinadas através de um conjunto de pluviômetros, colocados ao acaso ao lado da área útil das parcelas experimentais. As taxas de perdas de solo e a concentração de sedimentos foram obtidas pela pesagem do material coletado em potes plásticos com capacidade de $920 \mathrm{ml}$, colocados na extremidade inferior da calha coletora. As amostras foram coletadas durante
$60 \mathrm{~s}$, com intervalos de $3 \mathrm{~min}$ entre as coletas, os potes com solo e água foram pesados e, logo após, adicionados $5 \mathrm{ml}$ de sulfato de alumínio e potássio [KAl $\left(\mathrm{SO}_{4}\right)_{2} \cdot 12 \mathrm{H}_{2} \mathrm{O}$ ] a $5 \%$ para acelerar o processo de sedimentação. Os potes permaneceram em repouso por 24 horas e com auxílio de uma mangueira plástica, foi retirado por sifonamento a água sobrenadante. Posteriormente, os potes foram colocados em estufa a uma temperatura média de 50 a 60 ㄷ até peso constante. Em seguida os potes foram pesados com e sem o solo seco. A massa de água foi calculada, subtraindo-se a massa de solo perdida da massa total da enxurrada. A relação entre a massa de solo seco e a massa de mistura água-sedimento expressa a concentração de sedimentos em peso $\left(\mathrm{kg} \mathrm{l}^{-1}\right)$.

As perdas totais de solo em entressulcos foram obtidas pela Equação 1:

$$
\mathrm{PSi}=\frac{\sum \mathrm{QCt}}{\mathrm{A}}
$$

Em que: PSi é a perda de solo em entressulcos $\left(\mathrm{kg} \mathrm{m}^{-2}\right)$; Q é a descarga total $\left(\mathrm{kg} \mathrm{s}^{-1}\right)$; $C$ é a concentração de sedimentos na enxurrada $\left(\mathrm{kg} \mathrm{L}^{-1}\right)$; $\mathrm{t}$ é o intervalo de tempo entre duas amostragens (s); A é a área da parcela $\left(\mathrm{m}^{2}\right)$.

As taxas de desagregação do solo em entressulcos foram determinadas conforme a Equação 2:

$$
\mathrm{D}_{\mathrm{i}}=\frac{\mathrm{Mss}}{\mathrm{A} \mathrm{d} \mathrm{c}}
$$

Em que: $D_{i}$ é a taxa de desagregação do solo em entressulcos $\left(\mathrm{kg} \mathrm{m}^{-2} \mathrm{~s}^{-1}\right)$; Mss é a massa do solo seco desagregado ( $\mathrm{kg})$; A é a área da parcela $\left(\mathrm{m}^{2}\right)$; dc é a duração da coleta (s).

A erodibilidade nos entressulcos foi calculada pela Equação 3 (ELLIOT et al., 1989):

$$
\mathrm{K}_{\mathrm{i}}=\frac{\mathrm{D}_{\mathrm{i}}}{\mathrm{I}^{2} \mathrm{~S}_{\mathrm{f}}}
$$

Em que: $K_{i}$ é erodibilidade do solo em entressulcos $\left(\mathrm{kg} \mathrm{s} \mathrm{m}^{-4}\right) ; D_{i}$ é a taxa máxima de desagregação do solo nos entressulcos $\left(\mathrm{kg} \mathrm{m}^{-2} \mathrm{~s}\right.$ ${ }^{1}$ ), obtida através da média das quatro taxas mais consistentes de desagregação entre as últimas cinco observadas; I $^{2}$ é a intensidade da chuva ( $\mathrm{m} \mathrm{s}^{-}$ ${ }^{1}$ ), e $S_{f}$, o fator de correção para o declive dado pela equação de Liebenow et al. (1990).

A descarga líquida (qi), em $\mathrm{m}^{2} \mathrm{~s}^{-1}$, foi determinada a partir das coletas de enxurrada em potes plásticos, colocados na extremidade da 
calha de descarga, durante o tempo cronometrado, e dividido pela largura da parcela.

A velocidade superficial do escoamento $\left(V_{S}\right)$ foi determinada por meio da cronometragem do tempo gasto para que um corante, o azul de metileno, percorresse a distância entre dois pontos fixos na parcela. Essas determinações foram realizadas em intervalos de cinco minutos, a partir da formação da lâmina de escoamento. As velocidades médias do escoamento (Vm) foram determinadas pelo produto das velocidades superficiais do escoamento e um fator de correção $\alpha=2 / 3$, tendo em vista a distribuição do perfil de velocidade (KATZ et al., 1995).

Com base na determinação da descarga líquida (qi) e da velocidade média do escoamento (Vm) foram estimadas outras características hidráulicas: a altura da lâmina do escoamento (h) foi estimada através da equação derivada por Woolhiser e Liggett (1967) e Singh (1983); o número de Reynolds (Re) foi obtido pela metodologia proposta por Simons e Senturk (1992); o número de Froude (Fr) foi obtido por metodologia apresentada por Simons e Senturk (1992); a resistência ao escoamento foi determinada pelo fator de perda de carga $(f)$ de Darcy-Weisbach apresentada por Chow (1959); a rugosidade superficial foi determinada pelo coeficiente de Manning ( $n$ ) conforme Braida e Cassol (1999) e a tensão cisalhante do escoamento em entressulcos $(\tau)$ foi obtida por meio da metodologia proposta por Chow (1959).
Para a avaliação indireta dos efeitos causados pela formação de crosta e selamento superficial, foram repetidos os ensaios com chuvas simuladas em três repetições para cada solo, sendo determinada a taxa de desagregação e da erodibilidade do solo em entressulcos bem como a caracterização das variáveis hidráulicas do escoamento em entressulcos, seguindo a metodologia anteriormente citada, onde a superfície do solo não foi preparada e permaneceu exposta para secar ao ar durante em intervalos de 7 e 14 dias após a chuva inicial para formação de crosta após sua secagem, posteriormente comparadas com as determinações obtidas com a aplicação da chuva no tempo 0.

Os dados obtidos foram submetidos à análise de variância e as comparações múltiplas de médias foram realizadas pelo uso do Teste de Tukey a $5 \%$ de probabilidade. Foram utilizadas ainda, correlações simples e análises de regressão, com base no teste " $\mathrm{t}$ ". As análises foram realizadas por meio do uso do programa software R version 3.5.1 "Feather Spray" (R CORE TEAM, 2018).

\section{Resultados e Discussão}

Na Tabela 3 são apresentados os dados referentes às características hidráulicas do escoamento nos três períodos (0, 7 e 14 dias) em que foram aplicadas as chuvas simuladas. 
Tabela 3. Caracterização das variáveis hidráulicas do escoamento em entressulcos para os três ensaios com chuvas simuladas obtidas em condições de laboratório

\begin{tabular}{|c|c|c|c|c|c|}
\hline \multirow{3}{*}{ Variáveis } & \multirow{3}{*}{ Dias } & \multicolumn{4}{|c|}{ Classes de Solos $^{(1)}$} \\
\hline & & \multicolumn{2}{|c|}{ Mais desenvolvidos ${ }^{(2)}$} & \multicolumn{2}{|c|}{ Menos desenvolvidos ${ }^{(2)}$} \\
\hline & & LA & PV & RL & TC \\
\hline \multirow{2}{*}{$\mathbf{v}$} & 0 & $8,74 \mathrm{a}$ & $8,73 \mathrm{a}$ & $8,84 \mathrm{a}$ & 9,07 a \\
\hline & 7 & $8,83 \mathrm{a}$ & $8,68 \mathrm{a}$ & $8,66 \mathrm{a}$ & $9,02 \mathrm{a}$ \\
\hline $\mathrm{m}^{2} \mathrm{~s}^{-1} \times 10^{-7}$ & 14 & $8,73 \mathrm{a}$ & $8,80 \mathrm{a}$ & $8,71 \mathrm{a}$ & $9,01 \mathrm{a}$ \\
\hline \multirow{2}{*}{$\mathrm{Vm}$} & 0 & $0,03742 \mathrm{a}$ & $0,03873 a$ & $0,05071 b$ & $0,03691 b$ \\
\hline & 7 & $0,04342 \mathrm{a}$ & $0,03956 \mathrm{a}$ & $0,05678 a b$ & $0,04273 a b$ \\
\hline $\mathrm{m} \mathrm{s}^{-1}$ & 14 & 0,04315 a & $0,04143 \mathrm{a}$ & $0,05798 \mathrm{a}$ & $0,04460 \mathrm{a}$ \\
\hline \multirow{2}{*}{ qi } & 0 & $2,18 \mathrm{a}$ & $1,90 \mathrm{a}$ & $2,20 \mathrm{a}$ & $2,10 \mathrm{~b}$ \\
\hline & 7 & $2,21 \mathrm{a}$ & $1,95 \mathrm{a}$ & $2,19 \mathrm{a}$ & $2,17 a b$ \\
\hline $\mathrm{m}^{2} \mathrm{~s}^{-1} \times 10^{-5}$ & 14 & $2,25 \mathrm{a}$ & $1,96 \mathrm{a}$ & $2,17 \mathrm{a}$ & $2,24 a$ \\
\hline \multirow{2}{*}{ h } & 0 & $5,84 a$ & $5,01 \mathrm{a}$ & $4,33 \mathrm{a}$ & 5,69 a \\
\hline & 7 & $5,08 \mathrm{~b}$ & $4,97 \mathrm{a}$ & $3,86 \mathrm{a}$ & $5,09 a$ \\
\hline $\mathrm{m} \times 10-4$ & 14 & $5,20 a b$ & $4,76 \mathrm{a}$ & $3,75 \mathrm{a}$ & $5,01 \mathrm{a}$ \\
\hline \multirow{2}{*}{$\mathbf{n}$} & 0 & 0,05911 a & $0,05321 \mathrm{a}$ & $0,03570 \mathrm{a}$ & $0,05889 a$ \\
\hline & 7 & $0,04642 \mathrm{~b}$ & $0,05085 a$ & $0,02952 a$ & $0,04722 a b$ \\
\hline $\mathrm{s} \mathrm{m}^{-1 / 3}$ & 14 & $0,04746 a b$ & $0,04717 a$ & $0,02856 \mathrm{a}$ & $0,04477 \mathrm{~b}$ \\
\hline \multirow{2}{*}{$\mathbf{R e}$} & 0 & 24,99 a & $21,81 \mathrm{a}$ & 24,85 a & $23,17 b$ \\
\hline & 7 & $24,97 \mathrm{a}$ & $22,45 \mathrm{a}$ & 25,30 a & $24,11 a b$ \\
\hline Adimensional & 14 & $25,71 \mathrm{a}$ & $22,22 \mathrm{a}$ & $24,90 \mathrm{a}$ & 24,81 a \\
\hline \multirow{2}{*}{$\mathrm{Fr}$} & 0 & $0,49 a$ & $0,56 a$ & $0,78 \mathrm{~b}$ & 0,49 a \\
\hline & 7 & $0,62 \mathrm{a}$ & $0,57 \mathrm{a}$ & $0,92 \mathrm{a}$ & $0,60 \mathrm{a}$ \\
\hline Adimensional & 14 & $0,60 \mathrm{a}$ & $0,61 \mathrm{a}$ & $0,96 \mathrm{a}$ & $0,64 \mathrm{a}$ \\
\hline \multirow{2}{*}{$f$} & 0 & $3,28 \mathrm{a}$ & $2,90 \mathrm{a}$ & $1,31 \mathrm{a}$ & $3,28 \mathrm{a}$ \\
\hline & 7 & $2,12 b$ & $2,60 \mathrm{a}$ & $0,94 \mathrm{a}$ & $2,19 a b$ \\
\hline Adimensional & 14 & $2,20 a b$ & $2,27 \mathrm{a}$ & 0,89 a & $1,98 \mathrm{~b}$ \\
\hline \multirow{2}{*}{$\tau$} & 0 & $0,57 \mathrm{a}$ & 0,49 a & $0,42 \mathrm{a}$ & $0,56 \mathrm{a}$ \\
\hline & 7 & $0,50 \mathrm{~b}$ & $0,49 a$ & $0,38 a$ & $0,50 \mathrm{a}$ \\
\hline $\mathrm{Pa}$ & 14 & $0,51 a b$ & $0,47 a$ & $0,37 \mathrm{a}$ & $0,49 a$ \\
\hline
\end{tabular}

Fonte: ${ }^{(1)}$ De acordo com o Sistema Brasileiro de Classificação do Solo (EMBRAPA, 2013). Legenda: LA = Latossolo Amarelo; PV = Argissolo Vermelho; RL = Neossolo Litólico; $\mathrm{TC}=$ Luvissolo Crômico; $\mathrm{v}=$ viscosidade cinemática; $\mathrm{Vm}=$ velocidade média; $q i$ = descarga líquida unitária do escoamento; $\mathrm{h}=$ altura da lâmina de escoamento; $\mathrm{n}=$ coeficiente de rugosidade de Manning; $\mathrm{Re}=$ número de Reynolds; $\mathrm{Fr}=$ número de Froude; $f=$ fator de perda de carga; $\tau=$ tensão cisalhante do escoamento; ${ }^{(2)}$ Solos mais desenvolvidos: $\mathrm{Ki} \leq 2,46$; Solos menos desenvolvidos: $\mathrm{Ki}>2,46$. Médias seguidas de mesmas letras, nas colunas, não diferem entre si pelo teste de Tukey a $5 \%$ de probabilidade. 
O regime de escoamento em todos os solos foi caracterizado como laminar lento ou subcrítico, de acordo com os valores do número de Reynolds $(\operatorname{Re})<500$ e do número de Froude $(\mathrm{Fr})<1$ (Tabela 3), assim como os valores de descarga líquida (qi) na ordem de grandeza de $10^{-}$ ${ }^{5} \mathrm{~m}^{2} \mathrm{~s}^{-1}$ e altura de lâmina (h) $10^{-4} \mathrm{~m}$, juntamente com o regime de escoamento, caracterizado por baixos valores do número de Reynolds, indicam ocorrência de escoamento característico da erosão em entressulcos, semelhante ao observado por Cassol et al. (2004) e Cantalice et al. (2009).

É possível observar variações na velocidade média do escoamento $(\mathrm{Vm})$ apenas para o RL e o TC, em que a $\mathrm{Vm}$ tendeu a aumentar conforme o tempo entre os ensaios (Tabela 3). O Neossolo Litólico (RL) e o Luvissolo Crômico (TC) apresentaram diferenças estatísticas entre os períodos de aplicação dos testes com chuvas simuladas. A descarga líquida unitária do escoamento (qi) apresenta o mesmo padrão de comportamento observado para a Vm (Tabela 3), provavelmente por consequência dos efeitos do selamento superficial na fase úmida e da formação de crosta superficial após a secagem nos ensaios subsequentes. Para esta variável, é possível observar que somente no TC existe diferença significante entre os valores, sendo no ensaio aplicado aos 14 dias observada maior qi com relação as outras épocas. Provavelmente como consequência do aumento da $\mathrm{Vm}$ e do qi, observa-se a diminuição da altura da lâmina do escoamento (Tabela 3) nas diferentes épocas de aplicação das chuvas, com diferenças estatísticas significantes apenas no LA.

Esses fatos podem ser atribuídos possivelmente, mas não exclusivamente, aos altos teores da fração areia na composição textural dos solos avaliados, principalmente nos solos menos desenvolvidos ( $R L$ e TC), que apresentam cerca de $80 \%$ de areia total (Tabela 2 ), tendo maiores quantidades de partículas prontamente destacáveis, principalmente grãos de quartzo. Provavelmente, o material desagregado e disperso foi reorganizado e orientado superficialmente, ocorrendo à acomodação das partículas e seu rearranjo, o que possivelmente ocasionou a diminuição da rugosidade superficial e da sua resistência aos efeitos da enxurrada, aumentando a velocidade de escoamento, o que corrobora com o que foi observado em análise micromorfológica por Dalla Rosa et al. (2013), com auxílio de microscópio óptico em um Argissolo Vermelho-Amarelo, com $82 \%$ de areia em sua composição.

Observa-se na Tabela 3 a diminuição do coeficiente de rugosidade de Manning (n) e do fator de perda de carga $(f)$ a cada teste de simulação de chuva aplicada, havendo diferenças estatísticas entres os valores apenas para o LA e o TC. O coeficiente de rugosidade de Manning ( $n$ ) e o fator perda de carga $(f)$ representam o fator de resistência que a superfície do solo oferece ao deslocamento do escoamento, e sua diminuição indica, possivelmente, a homogeneização da superfície dos solos, devido ao processo de formação de crosta superficial (MAIER et al., 2013). Comportamento semelhante foi observado por Hu et al. (2012) que, avaliando o desenvolvimento de crostas superficiais no solo, em condições de chuva simulada, descrevem que o encrostamento superficial influencia na erosão do solo ao reduzir a rugosidade superficial, infiltração de água e erosão por salpicamento, ao mesmo tempo que aumenta o escoamento superficial.

Na Tabela 4, observa-se o comportamento da perda de solo (PSi), da taxa de desagregação do solo em entressulcos $\left(D_{i}\right)$ e da erodibilidade em entressulcos $\left(K_{i}\right)$ dos solos nos diferentes períodos de aplicação de chuva simulada, na qual verifica-se que $O P V$ e $O$ RL apresentaram maiores valores de PSi no primeiro ensaio (0 dias), diferindo estatisticamente do segundo e terceiro ensaios. Verifica-se ainda que não houve diferença estatística significante entres os valores de PSi, $D_{i}$ e $K_{i}$ apresentados no segundo ( 7 dias) e terceiro (14 dias) ensaios em nenhum dos solos avaliados (Tabela 4). Essa relação sinérgica entre $P S i, D_{i}$ e $K_{i}$ foi observada por Freitas et al. (2008) trabalhando com Luvissolos no semiárido, em que as perdas de solos, a taxa de desagregação e a erodibilidade do solo foram diretamente proporcionais. 
Tabela 4. Perdas totais de solo (PSi), taxa máxima de desagregação do solo em entressulcos $\left(D_{i}\right)$ e fator de erodibilidade do solo em entressulcos $\left(K_{i}\right)$, obtidos em condições de laboratório, sob chuva de $85 \mathrm{~mm} \mathrm{~h}^{-1}$ ( $\mathrm{i}$ $=2,36 \times 10^{-5} \mathrm{~m} \mathrm{~s}^{-1}$ ) e declividade de $0,10 \mathrm{~m} \mathrm{~m}^{-1} \mathrm{em}$ três épocas de aplicação

\begin{tabular}{|c|c|c|c|c|c|}
\hline \multirow{3}{*}{ Variáveis } & \multirow{3}{*}{ Dias } & \multicolumn{4}{|c|}{ Classes de Solos ${ }^{(1)}$} \\
\hline & & \multicolumn{2}{|c|}{ Mais desenvolvidos ${ }^{(2)}$} & \multicolumn{2}{|c|}{ Menos desenvolvidos ${ }^{(2)}$} \\
\hline & & LA & PV & $\mathbf{R L}$ & TC \\
\hline \multirow{2}{*}{ PSi } & 0 & $0,08840 \mathrm{a}$ & $0,19143 \mathrm{a}$ & $0,20850 a$ & $0,01777 \mathrm{a}$ \\
\hline & 7 & 0,29967 a & $0,04140 \mathrm{~b}$ & $0,02917 \mathrm{~b}$ & $0,01897 a$ \\
\hline $\mathrm{kg} \mathrm{m}^{-2}$ & 14 & $0,19400 \mathrm{a}$ & $0,02603 \mathrm{~b}$ & $0,01583 \mathrm{~b}$ & $0,01523 a$ \\
\hline \multirow{2}{*}{$D_{i}$} & 0 & $9,47 a$ & $9,77 \mathrm{a}$ & 9,26 a & $4,23 a$ \\
\hline & 7 & $5,00 \mathrm{~b}$ & $5,22 b$ & $4,67 b$ & $3,98 \mathrm{a}$ \\
\hline $\mathrm{kg} \mathrm{m}^{-2} \mathrm{~s}^{-1} \times 10^{-4}$ & 14 & $3,67 b$ & $3,74 \mathrm{~b}$ & $3,66 \mathrm{~b}$ & $3,69 a$ \\
\hline \multirow{2}{*}{$\mathbf{K}_{\mathbf{i}}$} & 0 & $3,54 \mathrm{a}$ & $3,69 \mathrm{a}$ & $3,41 \mathrm{a}$ & $1,60 \mathrm{a}$ \\
\hline & 7 & $1,89 \mathrm{~b}$ & $1,97 \mathrm{~b}$ & $1,75 b$ & $1,49 \mathrm{a}$ \\
\hline $\mathrm{kg} \mathrm{s} \mathrm{m}{ }^{-4} \times 10^{6}$ & 14 & $1,36 \mathrm{~b}$ & $1,39 \mathrm{~b}$ & $1,39 \mathrm{~b}$ & $1,38 \mathrm{a}$ \\
\hline
\end{tabular}

Fonte: ${ }^{(1)}$ De acordo com o Sistema Brasileiro de Classificação do Solo (EMBRAPA, 2013). Legenda: LA = Latossolo Amarelo; $\mathrm{PV}=$ Argissolo Vermelho; $\mathrm{RL}=$ Neossolo Litólico; $\mathrm{TC}=$ Luvissolo Crômico; $\mathrm{D}_{\mathrm{i}}=$ taxa de desagregação do solo em entressulcos; $K_{i}=$ erodibilidade em entressulcos. ${ }^{(2)}$ Solos mais desenvolvidos: $K i \leq 2,46$; Solos menos desenvolvidos: $\mathrm{Ki}>2,46$. Médias seguidas das mesmas letras minúsculas nas colunas não diferem entre si pelo teste de Tukey a $5 \%$ de probabilidade.

De forma geral, o TC apresentou comportamento diferenciado dos demais solos, mesmo apresentando em sua composição textural cerca de $80 \%$ de areia total. 0 alto teor de carbono orgânico e óxidos de ferro e alumínio em sua composição, atuando como agentes cimentantes, podem ter influenciado diretamente para um alto índice de estabilidade dos agregados (Tabela 2), conferindo resistência a desagregação, apresentando os menores valores para PSi, $D_{i}$ e $K_{i}$ (Tabela 4), e comportamento estável, sem alterações drásticas entre as aplicações de chuva simulada Lado e Ben-Hur (2004) afirmam que em solos com teores elevados de quartzo e feldspato (partículas que apresentam cargas superficiais próximas a zero) a estabilidade dos agregados é controlada principalmente por outros agentes, como matéria orgânica e óxidos de ferro e alumínio, o que pode explicar o comportamento do Luvissolo Crômico (TC) avaliado neste estudo.

$\mathrm{Na}$ Figura 1, é possivel observar a evolução das modificações causadas pelos eventos de chuva simulada na rugosidade superficial do solo, pelo processo contínuo e simultâneo de destacamento, transporte e sedimentação de partículas ao longo do tempo de precipitação. Em cada um dos solos avaliados, nota-se comportamento semelhante durante a aplicação do primeiro evento de chuva simulada (0 dias), no qual ocorre a redução na rugosidade superficial do solo $\left(R^{2}\right.$ entre $0,73-0,93$ e $p<0,01$ em todos os solos), indicando a ocorrência selamento superficial e todos os processos envolvidos, levando a uniformidade do micro relevo e a alteração nas caracteristicas hidráulicas do escoamento discutidas anteriormente, pela redução das forças de resistência do solo ao escoamento. 
Figura 1. Evolução da rugosidade superficial de Manning (n) no Latossolo Amarelo (LA), Argissolo Vermelho (PV), Neossolo Litólico (RL) e Luvissolo Crômico (TC) ao longo da duração de aplicação das chuvas nos tempos de ensaio 0,7 e 14 dias
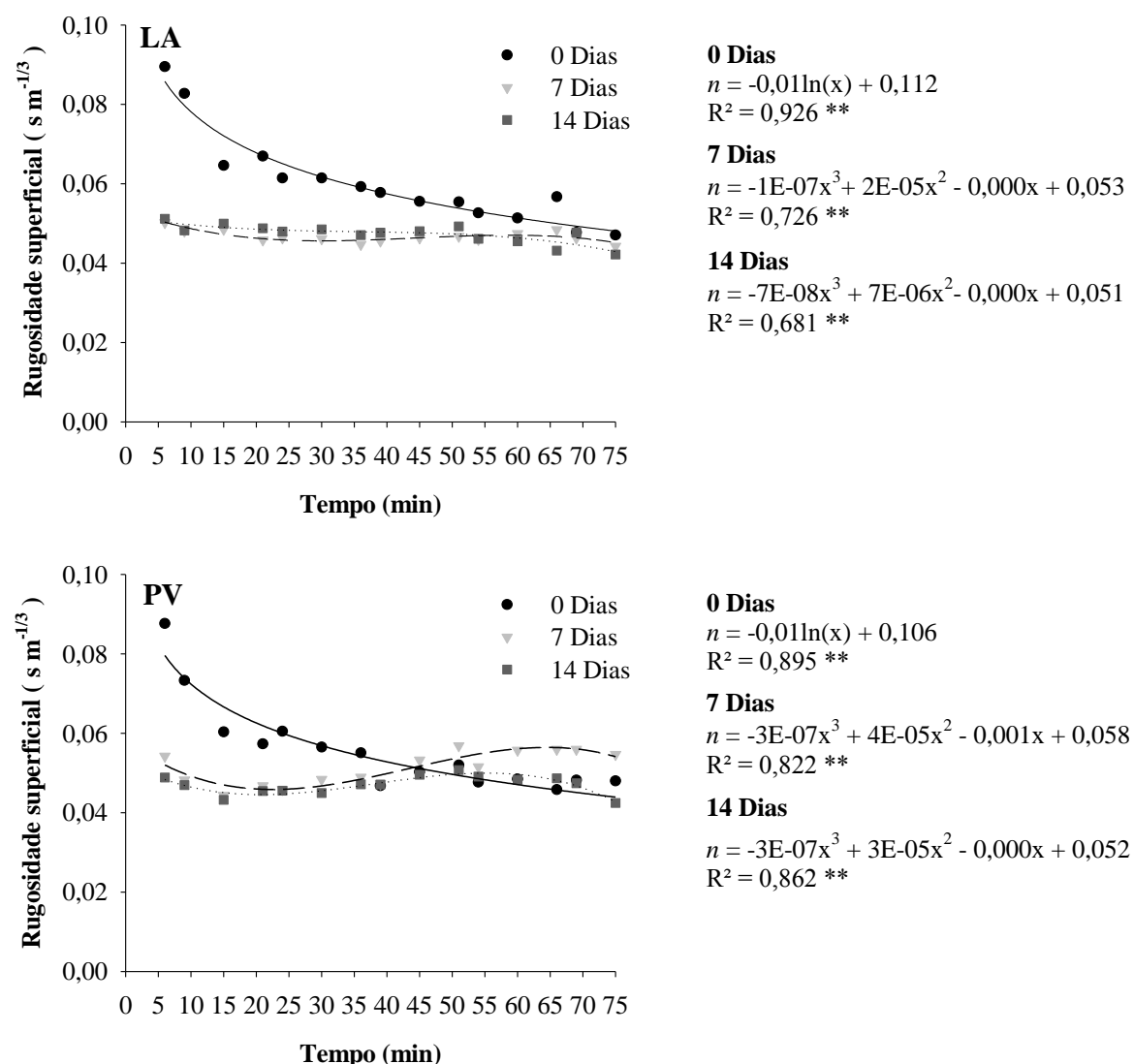

0 Dias

$n=-0,01 \ln (\mathrm{x})+0,106$

$\mathrm{R}^{2}=0,895 * *$

7 Dias

$n=-3 \mathrm{E}-07 \mathrm{x}^{3}+4 \mathrm{E}-05 \mathrm{x}^{2}-0,001 \mathrm{x}+0,058$ $\mathrm{R}^{2}=0,822 * *$

14 Dias

$n=-3 \mathrm{E}-07 \mathrm{x}^{3}+3 \mathrm{E}-05 \mathrm{x}^{2}-0,000 \mathrm{x}+0,052$ $\mathrm{R}^{2}=0,862 * *$

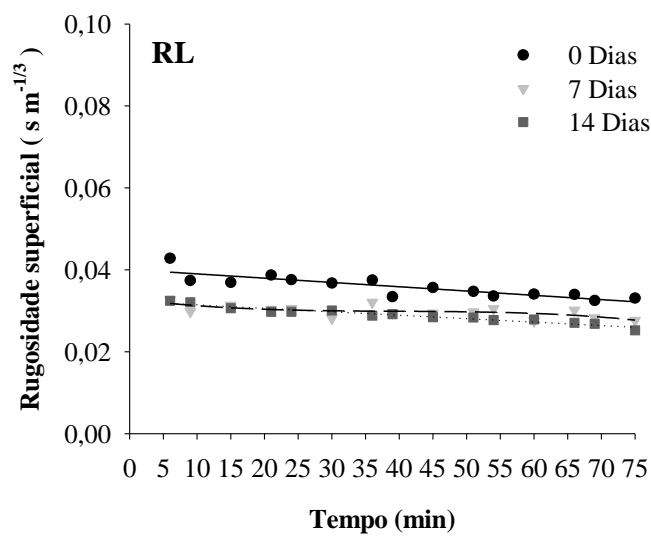

0 Dias

$n=-0,000 \mathrm{x}+0,040$

$\mathrm{R}^{2}=0,732 * *$

7 Dias

$n=-4 \mathrm{E}-08 \mathrm{x}^{3}+5 \mathrm{E}-06 \mathrm{x}^{2}-0,000 \mathrm{x}+0,032$ $\mathrm{R}^{2}=0,445 * *$

14 Dias

$n=-8 \mathrm{E}-05 \mathrm{x}+0,032$

$\mathrm{R}^{2}=0,935 * *$

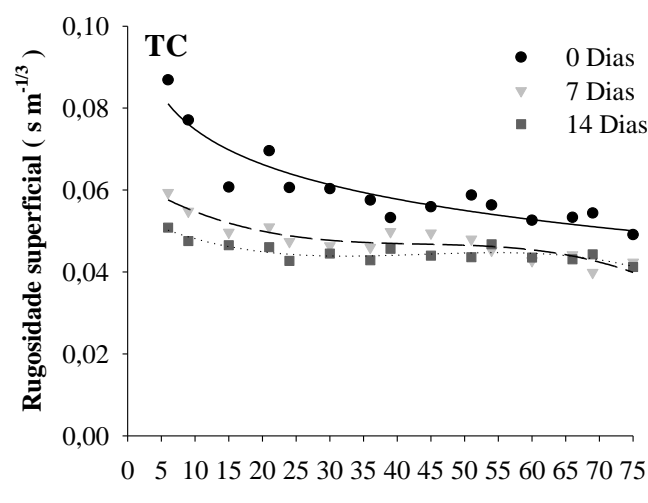

0 Dias

$n=-0,01 \ln (\mathrm{x})+0,103$

$\mathrm{R}^{2}=0,855 * *$

7 Dias

$n=-2 \mathrm{E}-07 \mathrm{x}^{3}+2 \mathrm{E}-05 \mathrm{x}^{2}-0,001 \mathrm{x}+0,063$ $\mathrm{R}^{2}=0,837 * *$

14 Dias

$n=-2 \mathrm{E}-07 \mathrm{x}^{3}+2 \mathrm{E}-05 \mathrm{x}^{2}-0,000 \mathrm{x}+0,054$

$\mathrm{R}^{2}=0,776 * *$

Tempo (min) 
Os solos avaliados apresentaram comportamentos semelhantes quando analisados o segundo e o terceiro eventos de chuvas simuladas (aos 7 e 14 dias) como pode ser observado na Figura 1 (confirmando comportamento das médias apresentadas na Tabela 4), sendo os maiores valores para o coeficiente de ajuste do modelo $\left(R^{2}\right)$ obtidos através da utilização do modelo polinomial de ordem 3, ou polinomial cúbico, com exceção do $\mathrm{RL}$, que, para o seu comportamento no evento de chuva simulada aplicada aos 14 dias, o modelo linear foi o mais adequado.

Possivelmente, o modelo polinomial cúbico, reflete de maneira mais adequada $o$ comportamento dos eventos de destacamento, transporte e sedimentação que ocorrem durante processo de erosão hídrica, após a formação de uma crosta superficial, devido a ser apresentado em ordens que representam modificações no sentido do comportamento na tendência das curvas. Por esses eventos ocorrerem de forma simultânea e contínua durante os eventos de precipitação, mesmo partículas que já foram destacadas, transportadas e sedimentadas, podem, ao longo do tempo e do espaço (comprimento da faixa de solo), serem novamente destacadas, transportadas e sedimentadas em outro ponto do terreno (ZHANG et al., 2017; ZHANG; WANG, 2017) causando efeito gráfico visualizado em forma de onda senoidal.

O desenvolvimento do selo pode ser contínuo, e um equilíbrio dinâmico entre a destruição e a formação do selo pode ser estabelecido (ARMENISE et al., 2018). Observa-se na Figura 1 que ocorrem modificações na rugosidade superficial do solo ao longo do tempo de duração da chuva, demonstrando que ocorre a quebra da crosta e posterior realocação das partículas de solo em outros pontos do espaço, formando selamento e posterior crosta em outro ponto, onde possivelmente já ocorreu o mesmo fenômeno

De Roo e Riezebos (1992) afirmam que, quando se aplicam precipitações pluviais sucessivas ao solo, a maior porção da camada de encrostamento se desenvolve logo com a aplicação inicial; o efeito das chuvas subsequentes é menos relevante no decréscimo da taxa de infiltração e nas variáveis hidráulicas do escoamento, 0 que se assemelha aos resultados encontrados neste experimento (Tabela 4 e Figura 1). Comportamento semelhante também foi observado por Zonta et al. (2012), em que os autores constataram a formação do encrostamento superficial já com a primeira aplicação de chuva, exercendo papel fundamental no comportamento das variáveis hidráulicas do escoamento e na infiltração nos ensaios subsequentes.

\section{Conclusões}

A formação de selamento e a existência de crosta superficial foram observadas, de forma indireta, no Latossolo Amarelo (LA), Argissolo Vermelho (PV) e no Neossolo Litólico (RL), devido ao aumento da velocidade média de escoamento e da descarga liquida, diminuição da altura da lâmina de água, bem como as perdas totais de solo (PSi), taxa máxima de desagregação $\left(D_{i}\right)$ e o fator de erodibilidade em entressulcos $\left(\mathrm{K}_{\mathrm{i}}\right)$;

A maior contribuição para formação do encrostamento superficial ocorre no primeiro evento de chuva simulada (dia 0 ), sendo o efeito das chuvas subsequentes menos significativas nas perdas totais de solo, nas taxas de desagregação e erodibilidade do solo em entressulco para o Latossolo Amarelo (LA), o Argissolo Vermelho (PV) e o Neossolo Litólico (RL);

Após a formação de crosta superficial, eventos de precipitação subsequentes tendem a resultar em equilíbrio dinâmico entre a destruição e a formação do selo superficial de forma contínua ao longo da duração das chuvas;

A avaliação da modificação das caracteristicas hidráulicas do escoamento superficial, das taxas de desagregação e do fator de erodibilidade do solo em entressulcos se mostram viáveis para a observação do fenômeno de formação de selo e de crosta superficial em solos com caracteristicas semelhantes aos avaliados nesse estudo.

\section{Referências}

AGÊNCIA EXECUTIVA DE GESTÃO DAS ÁGUAS. Meteorologia: chuvas. Paraíba, PB: AESA, 2019. Disponível em:

https://www.aesa.pb.gov.br/aesa-

website/meteorologia-chuvas/climatologia/.

Acesso em: 08 fev. 2019.

ARMENISE, E.; SIMMONS, R. W.; AHN, S.; GARBOUT, A.; DOERR, S. H.; MOONEY, S. J.; STURROCK, C. J.; RITZ, K. Soil seal development under simulated rainfall: structural, physical and hydrological dynamics. Journal of Hydrology, 
v.556, p. 211-219, 2018.

https://doi.org/10.1016/i.jhydrol.2017.10.073

ASSOULINE, S. Rainfall-induced soil surface sealing: a critical review of observations, conceptual models and solutions. Vadose Zone Journal, v.3, n.2, p. 570-591, 2004.

https://doi.org/10.2136/vzj2004.0570

BRAIDA, J. A.; CASSOL, E. A. Relações de erosão em entressulcos com o tipo e a quantidade de resíduo vegetal na superfície do solo. Revista Brasileira de Ciência do Solo, v.23, n.3, p. 711721 1999. http://dx.doi.org/10.1590/S010006831999000300026.

BRANDÃO, V. S.; SILVA, D. D.; RUIZ, H. A.; PRUSKI, F. F.; SCHAEFER, C. E. G. R.; MARTINEZ, M. A.; MENEZES, S. J. M. C. Resistência hidráulica da crosta formada em solos submetidos a chuvas simuladas. Revista Brasileira de Ciência do Solo, v.30, n.1, p. 13-21, 2006.

http://dx.doi.org/10.1590/S0100-

$\underline{06832006000100002}$.

BRASIL. Ministério da Agricultura. I. Levantamento exploratório de reconhecimento dos solos do Estado da Paraíba. II Interpretação para uso agrícola dos solos do Estado da Paraíba. Rio de Janeiro: Sudene, 1972. 683 p. (Boletim Técnico, 15; SUDENE-DRN. Série Pedologia, 8).

CANTALICE, J. R. B.; BEZERRA, S. A.; OLIVEIRA, O. F. L.; MELO, R. O. Hidráulica e taxas de erosão em entressulcos sob diferentes declividades e doses de cobertura morta. Revista Caatinga, v.22, n.2, p. 68-74, 2009.

CARMI, G; BERLINER, P. The effect of soil crust on the generation of runoff on small plots in an arid environment. Catena, v.74, n.1, p. 37-42, 2008. https://doi.org/10.1016/i.catena.2008.02.002

CASSOL, E. A.; CANTALICE, J. R. B.; REICHERT, J. M.; MONDARDO, A. Escoamento superficial e desagregação do solo em entressulcos em solo franco-argilo-arenoso com resíduos vegetais. Pesquisa Agropecuária Brasileira, v.39, n.7, p. 685-690, 2004.

http://dx.doi.org/10.1590/S0100-

$\underline{204 \times 2004000700010}$
CASTRO FILHO, C.; MUZILLI, O.; PODANOSCHI, A. L. Estabilidade dos agregados e sua relação com o teor de carbono orgânico num Latossolo Roxo Distrófico, em função de sistemas de plantio, rotações de culturas e métodos de preparo das amostras. Revista Brasileira de Ciência do Solo, v.22, n.3, p. 527-538, 1998.

http://dx.doi.org/10.1590/S0100$\underline{06831998000300019}$.

CESÁRIO, F. V.; DONAGEMMA, G. K.; RUIZ, H. A.; BALIEIRO, F. C. Estabilidade de agregados em água: análise crítica e padronização. Rio de Janeiro: Embrapa Solos, 2010. 7 p. (Série Embrapa Solos. Comunicado técnico, 57).

CHOW, V. T. Open channel hydraulics. New York: McGraw-Hill. 1959. 690 p.

DALLA ROSA, J.; COOPER, M.; DARBOUX, F.; MEDEIROS, J.C. Processo de formação de crostas superficiais em razão de sistemas de preparo do solo e chuva simulada. Revista Brasileira de Ciência do Solo, v.37, n.2, p. 400-410, 2013.

http://dx.doi.org/10.1590/S0100-

$\underline{06832013000200011}$

DE ROO, A. P. J.; RIEZEBOS, H. T. Infiltration experiments on loess soils and their implications for modelling surface runoff and soil erosion. Catena, v.19, n.2, p. 221-239, 1992. https://doi.org/10.1016/0341-8162(92)90026-8

ELLIOT, W. J.; LIEBNOW, A. M.; LAFLEN, J. M.; $\mathrm{KOHL}, \mathrm{K}$. D. A compendium of soil erodibility data from WEPP cropland soil field erodibility experiments $1987 \& 88$. West Lafayette: The Ohio State University and USDA-ARS, 1989. 316 p.

EMBRAPA. Sistema Brasileiro de Classificação de Solos. 3. ed. Brasília, DF: Embrapa, 2013. 353 p.

FARIAS, D. R.; OLIVEIRA, F. H. T.; SANTOS, D.; ARRUDA, J. A.; HOFFMANN, R. B.; NOVAIS, R. F. Fósforo em solos representativos do Estado da Paraíba. I. Isotermas de adsorção e medidas do fator capacidade de fósforo. Revista Brasileira de Ciência do Solo, v.33, n.3, p. 623-632, 2009. http://dx.doi.org/10.1590/S0100$\underline{06832009000300015}$ 
FOX, D. M.; LE BISSONNAIS, Y. A process-based analysis of the influence of aggregate stability on surface crusting, infiltration, and interrill erosion. Soil Science Society of America Journal, v.62, n.3, p. 717-724, 1998.

http://doi.org/10.2136/sssaj1998.036159950062 $00030025 x$

FRANCISCO, P. R. M.; MEDEIROS, R. M.; SANTOS, D.; MATOS, R. M. Classificação Climática de Köppen e Thornthwaite para o Estado da Paraíba.

Revista Brasileira de Geografia Física, v.8, n.4, p. 1006-1016, 2015. https://doi.org/10.5935/1984$\underline{2295.20150049}$

FREITAS, F. J.; CANTALICE, J. R. B.; BEZERRA, S. A.; OLIVEIRA, M. D. R.; LIMA, P. A.; FIGUEIRA, S. B. Erosão em entressulcos sob caatinga e culturas agrícolas. Revista Brasileira de Ciência do Solo, v.32, n.4, p. 1743-1751, 2008.

http://dx.doi.org/10.1590/S0100-

$\underline{06832008000400039}$

FREITAS, T. S. Atributos físicos de solos representativos do Estado da Paraíba. 2016. $61 \mathrm{f}$. Dissertação (Mestrado em Ciência do Solo) Universidade Federal da Paraíba, 2016.

HILLEL, D. Introduction to environmental soil physics. Burlington: Academic Press, 2003. p. 7389. https://doi.org/10.1016/B978-0123486554/50006-X

HU, X.; LIU, L. Y.; LI, S. J.; CAI, Q. G.; LÜ, Y. L.; GUO, J. R. Development of soil crusts under simulated rainfall and crust formation on a loess soil as influenced by polyacrylamide. Pedosphere, v.22, n. 3 p. 415-424, 2012.

https://doi.org/10.1016/S1002-0160(12)60027-7

KATZ, D. M.; WATTS, F. J.; BURROUGHS, E. R. Effects of surface roughness and rainfall impact on overland flow. Journal Hydrology Division, v.121, n.7, p. 546-553, 1995.

http://doi.org/10.1061/(ASCE)0733-

$\underline{\text { 9429(1995)121:7(546) }}$

KINNELL, P. I. A. Raindrop impact induced erosion processes and prediction: a review. Hydrological Processes, v.19, n.14, p. 2815-2844, 2005.

http://doi.org/10.1002/hyp.5788
LADO, M.; BEN-HUR, M. Soil mineralogy effects on seal formation, runoff and soil loss. Applied Clay Science, v.24, n.3-4, p. 209-224, 2004. https://doi.org/10.1016/j.clay.2003.03.002

LE BISSONNAIS, Y.; CERDAN, O.; LECOMTE, V.; BENKHADRA, H.; SOUCHERE, V.; MARTIN, P. Variability of soil surface characteristics influencing runoff and interrill erosion. Catena, v.62, n.2-3, p. 111-124, 2005.

http://doi.org/10.1016/j.catena.2005.05.001

LE BISSONNAIS, Y.; SINGER, M. J. Seal formation, runoff and interrill erosion from 17 California soils. Soil Science Society of America Journal, v.57, n.1, p. 224-229, 1993.

http://doi.org/10.2136/sssaj1993.036159950057 $\underline{00010039 x}$

LIEBENOW, A. M.; ELLIOT, W. J.; LAFLEN, J. M.; $\mathrm{KOHL}, \mathrm{K} . \mathrm{O}$. Interrill erodibility: collection and analysis of data from cropland soils. Transactions of the American Society of Agricultural Engineers, v.33, n.6 p. 1882-1888, 1990.

http://doi.org/10.13031/2013.31553

MAIER, C.; KAUFMANN, V.; MERTEN, G. H.; CASTRO, N. M. R.; OLIVEIRA, F. P. Efeito da crosta superficial na erosão entressulcos de um Neossolo Litólico submetido a diferentes usos e manejo. Revista Brasileira de Recursos Hídricos, v.18, n.1, p. 149-163, 2013.

http://doi.org/10.21168/rbrh.v18n1.p149-163

MEYER, L. D.; HARMON. W. C. Multiple intensity rainfall Simulator for erosion research on row sideslopes. Transactions of the American Society of Agricultural Engineers, v.22, n.1, p. 100-103, 1979. http://doi.org/10.13031/2013.34973

MONTANARELLA, L.; PENNOCK, D. J.; MCKENZIE, N.; BADRAOUI, M.; CHUDE, V.; BAPTISTA, I.; MAMO, T.; YEMEFACK, M.; SINGH AULAKH, M.; YAGI, K.; YOUNG HONG, S.; VIJARNSORN, P.; ZHANG, G.-L.; ARROUAYS, D.; BLACK, H.; KRASILNIKOV, P.; SOBOCKÁ, J.; ALEGRE, J.; HENRIQUEZ, C. R.; DE LOURDES MENDONÇASANTOS, M.; TABOADA, M.; ESPINOSA-VICTORIA, D.; ALSHANKITI, A.; ALAVIPANAH, S. K.; ELSHEIKH, E. A. E. M.; HEMPEL, J.; CAMPS ARBESTAIN, M.; NACHTERGAELE, F.; VARGAS, R. World's soils are under threat. Soil, v.2, p. 79-82, 2016.

https://doi.org/10.5194/soil-2-79-2016 
OLIVEIRA, F. H. T.; LEAL, J. V.; SANTOS, D.; FARIAS, D. R.; ARRUDA, J. A. Banco de solos representativos do Estado da Paraíba. In: REUNIÃO BRESILEIRA DE MANEJO E CONSERVAÇÃO DO SOLO E DA ÁGUA (RBMCSA), 16., 2006, Aracaju. Anais [...]. Aracaju: SBCS, 2006. 1 CD-ROM.

PEQUENO, P. L. L. Funções de pedotransferência para estimativa de retenção de água em solos da mesorregião do agreste paraibano. 2016. $188 \mathrm{f}$. Tese (Doutorado em Ciência do Solo) Universidade Federal da Paraíba, 2016.

R CORE TEAM: a language and environment for statistical computing. Version 3.5.1. Vienna: R Foundation for Statistical Computing, 2018. Disponível em: https://www.r-project.org/. Acesso em: 24 ago. 2018.

SANTOS, H. C.; OLIVEIRA, F. H. T.; SALCEDO, I. H.; SOUZA, A. P.; SILVA, V. D. M. Kinetics of phosphorus sorption in soils in the state of Paraíba. Revista Brasileira de Ciência do Solo, v.35, n.4 p. 1301-1310, 2011.

http://dx.doi.org/10.1590/S0100-

06832011000400024

SIMONS, D. B.; SENTÜRK, F. Sediment transport technology: water and sediment dynamics. Littleton: Water Resources Publication, 1992. 897 p.

SINGH, V. P. Analytical solutions of cinematic for erosion on a plane: II Rainfall of finite duration. Advanced Water Research, v.6, n.2, p. 88-95, 1983. https://doi.org/10.1016/03091708(83)90045-3

SOUZA, E. S.; ANTONINO, A. C. D.; HECK, R. J.; MONTENEGRO, S. M. G. L.; LIMA, J. R. S.; SAMPAIO, E. V. S. B.; ANGULO-JARAMILLO, R.; VAUCLIN, M. Effect of crusting on the physical and hydraulic properties of a soil cropped with Castor beans (Ricinus communis L.) in the northeastern region of Brazil. Soil and Tillage Research, v.141, n.8, p. 55-61, 2014. https://doi.org/10.1016/j.still.2014.04.004

TEIXEIRA, P. C.; DONAGEMMA, G. K.; FONTANA, A.; TEIXEIRA, W. G. (Ed.). Manual de métodos de análise de solo. 3.ed. Brasília: Embrapa, 2017. $573 \mathrm{p}$.
TOUMA, J.; RACLOT, D.; AL-ALI, Y.; ZANTE, P.; HAMROUNI, H.; DRIDI, B. In situ determination of the soil surface crust hydraulic resistance. Journal of Hidrology, v.403, n.3, p. 253-260, 2011. https://doi.org/10.1016/i.jhydrol.2011.04.004

VAEZI, A. R.; BAHRAMI, H. A. Relationship between soil productivity and erodibility in rainfed wheat lands in northwestern Iran. Journal of Agricultural Science and Technology, v.16, n.3 p. 1455-1466, 2014.

https://doi.org/10.2478/v10247-012-0097-4

WOOLHISER, D. A.; LIGGETT, J. A. Unsteady, onedimensional flow over a plane - The rising hydrograph. Water Resources Research, v.3, n.3 p. 753-771, 1967.

https://doi.org/10.1029/WR003i003p00753

ZHANG, X. C.; NEARING, M. A.; GARBRECHT, J. D. Gaining insights into interrill erosion processes using rare earth element tracers. Geoderma, v.299, n.1, p. 63-72, 2017.

https://doi.org/10.1016/i.geoderma.2017.04.004

ZHANG, X. C.; WANG, Z. L. Interrill soil erosion processes on steep slopes. Journal of Hydrology, v.548, n.5, p. 652-664, 2017.

https://doi.org/10.1016/j.jhydrol.2017.03.046

ZONTA, J. H.; MARTINEZ, M. A.; PRUSKI, F. F.; SILVA, D. D.; SANTOS, M. R. Efeito da aplicação sucessiva de precipitações pluviais com diferentes perfis na taxa de infiltração de água no solo. Revista Brasileira de Ciência do Solo, v.36, n.2, p. 377-388, 2012. http://dx.doi.org/10.1590/S010006832012000200007 